\title{
Cardiovascular risk assessment in the senior population undergoing anesthesia for non-cardiac surgery
}

\author{
Concezione Tommasino
}

Department of Biomedical, Surgical and Odontoiatric Sciences, University of Milan, Italy

\begin{abstract}
Older patients are underrepresented in major cardiovascular trials, and only relatively healthy elderly patients, with few comorbidities or functional impairments, have been enrolled. As a result, current guidelines are unable to provide evidence-based recommendations for anesthesia treatment of patients aged $\geq 75$ years, undergoing non-cardiac surgical procedures. Effective strategies, aimed at reducing the risk of perioperative cardiac complications, should involve cardiac evaluation using mostly medical history. A key component is the evaluation of active or unstable cardiac conditions, surgical and cardiac risk factors, and functional capacity of the patient.

Patient at low cardiac risk, based on clinical features, functional status, and low-risk surgery, do not generally require further cardiac evaluation, and can be operated on safely without further delay. Additional preoperative testing is indicated in patients at intermediate risk, with poor or unclear functional status. Patients at high-risk based on clinical features, poor functional status, undergoing high-risk surgery may benefit from further evaluation with noninvasive/invasive stress testing. In case of emergency surgical procedures, patient or surgeryspecific factors dictate the strategy and do not allow further cardiac testing or treatment.

Successful perioperative evaluation is best achieved by combining an integrated multidisciplinary approach, with good communication between the patient, anesthesiologist, cardiologist, geriatrician and surgeon.
\end{abstract}

Corresponding author: Concezione Tommasino, Department of Biomedical, Surgical and Odontoiatric Sciences, Università degli Studi di Milano, Polo Universitario ASST Santi Paolo e Carlo, Via di Rudinì 8, 20142 Milano, Italy. E-mail: concezione.tommasino@unimi.it

Key words: Elderly patient; cardiac risk; surgical risk; non-cardiac surgery; functional capacity; non invasive cardiac testing; invasive cardiac testing.

Received for publication: 26 April 2017

Accepted for publication: 4 May 2017

(C) Copyright C. Tommasino, 2017

Tipografia PI-ME Editrice, Italy

Monaldi Archives for Chest Disease 2017; 87:853

doi: 10.4081/monaldi.2017.853

This article is distributed under the terms of the Creative Commons Attribution Noncommercial License (by-nc 4.0) which permits any noncommercial use, distribution, and reproduction in any medium, provided the original author(s) and source are credited.

\section{Introduction}

"The strongest predictors for postoperative mortality and morbidity are preoperative variables"

The incidence and prevalence of most cardiovascular disorders increase with age. It is estimated from primary care data that, in the 7584 years age group, $19 \%$ of men and $12 \%$ of women have some degree of cardiovascular disease (CVD) (1), and CVD is the leading cause of death and major disability in adults $\geq 75$ years of age. Age per se, is responsible for only a small increase in the risk of perioperative complications; greater risks are associated with urgency of the surgical procedure and the presence of comorbidities. Unfortunately, older patients have been markedly underrepresented in most major cardiovascular trials or the studies have enrolled only relatively healthy older patients with few comorbidities or functional impairments. As a result, current guidelines are unable to provide evidence-based recommendations for anesthesia treatment of patients aged $\geq 75$ years [2]. The aim of guidelines is to help physicians to make safe decisions and formulate a management plan by using the latest evidence-based medical knowledge and simplify and/or eliminate unnecessary employment of resources.

In 2015, the American Heart Association, the American College of Cardiology, and the American Geriatrics Society in their statement concluded that only the results of large population-based studies and clinical trials, that include older patients representative of those seen in clinical practice, will provide the foundation for future evidence-based guidelines applicable to older people with CVD [2].

Notwithstanding these considerations, although there are no specific guidelines for the senior population, we can refer to guidelines and recommendations based on available relevant clinical evidence that can help the anesthesia care providers to assess the preoperative cardiovascular risk in adult patients and to design perioperative strategies that aim to reduce additional perioperative risks [3-6].

\section{Anesthesiologic approach}

Preoperative risk assessment is an important step in reducing perioperative morbidity and mortality in patients undergoing noncardiac surgery.

Effective strategies, aimed at reducing the risk of perioperative cardiac complications, should mostly involve cardiac evaluation using medical history, before the surgical procedure. A key component is the evaluation of active or unstable cardiac conditions, surgical and cardiac risk factors, and functional capacity of the patient. Successful perioperative evaluation is best achieved by combining an integrated multidisciplinary approach, with good communication between the patient, anesthesiologist, cardiologist, geriatrician and surgeon.

Anesthesiologists, who are experts on the specific demands of the proposed surgical procedure, need to assess the cardiac risk associated 
with the surgery, identify the patient risk profile, recommend appropriate preoperative testing and make suitable recommendations.

Cardiac risk estimation is initially based on clinical characteristics and type of surgery, and then extended, when indicated, to resting electrocardiography (ECG), laboratory measurements, and non-invasive/invasive stress testing.

\section{Assess the clinical features}

Before discussing how to evaluate the cardiovascular risk in the senior population, it is mandatory to underlye that the preoperative anesthesiologic assessment of geriatric patients needs to include all perioperative risk factors such as frailty, functional status, nutritional status, pulmonary status, and substance dependence [7,8]. The risk of perioperative cardiac complications depends on the cardiac conditions of the patient before surgery, the prevalence of comorbidities, and the urgency, magnitude, type, and duration of the surgical procedure. Factors that increase the risk of perioperative cardiac complications include: ischemic heart disease, heart failure, diabetes mellitus (especially insuline-requiring), renal insufficiency, poor functional status, and high risk surgery.

\section{Consider the urgency of the procedure}

When emergency surgical procedures, such as those for ruptured abdominal aortic aneurysm, major trauma, or perforated viscus are needed, the cardiac evaluation must necessarily be limited. Patient or surgery-specific factors dictate the strategy and do not allow further cardiac testing or treatment. In these cases, the consultant provides recommendations on perioperative medical management, surveillance for cardiac events, and continuation of chronic cardiovascular medical therapy [6]. In urgent procedures, intraoperative hemodynamic monitoring should always be planned.

\section{Consider the surgical risk factors}

In order to stratify overall perioperative risk, it is essential to consider the nature and duration of the surgical procedure. Although patient-specific factors are more important than surgery-specific factors in predicting the cardiac risk for non-cardiac surgical procedures, the type of surgery must be taken into consideration. In the majority of the cases, the surgical procedure itself represents a sustained cardiovascular stress, quite beyond what the patient may experience in daily life. The risk of perioperative cardiac complications depends mostly on surgical procedures associated with prolonged haemodynamic and cardiac stress, as well as perioperative changes in body core temperature, blood loss, fluid shifts, and whatsoever stress that increases myocardial oxygen demand.

Table 1 illustrates surgical risk estimates (low, intermediate, and high-risk groups), considering the combined incidence at 30-days of cardiac death and nonfatal myocardial infarction, according to type of surgery or intervention (patients' comorbidities are not considered) [9].

\section{Evaluate functional capacity}

Determination of functional capacity is a pivotal step in pre-operative cardiac risk assessment, and represents the ability to climb stairs, do one's own housework, perform regular exercise, etc. Functional capacity can be measured in metabolic equivalents (METs), and can be used as a predictor of future cardiac events. One MET equals the basal metabolic rate (oxygen consuption at rest $=3.5 \mathrm{ml} \mathrm{O}_{2} / \mathrm{kg} \mathrm{bw} / \mathrm{min}$ ), and exercise testing provides an objective assessment of functional capacity.

Without testing, however, functional capacity can be estimated from the ability to perform daily living activities. Walking on level ground (about $6 \mathrm{~km} / \mathrm{h}$ ) or climbing two flights of stairs demands 4 METs, while strenuous sports, such as swimming, $>10$ METS. Generally, <4 METs indicates poor functional capacity, and is associated with an increased incidence of post-operative cardiac events [10] and worse short- and long-term outcome in patients undergoing noncardiac surgery.

\section{Calculate risk indices}

Clinical risk indices are recommended for perioperative risk stratification [3-6]. Several risk indices have been developed during the past 30 years. At the moment, the Lee risk index (6 predictors: type of surgery, ischemic heart disease, heart failure, cerebrovascular disease, treatment with insulin, and creatinine ( $>2 \mathrm{mg} / \mathrm{dL}$ ), a modified version of the original Goldman index; and the NSQIP model (5 predictors: age, ASA physical status class, creatinine ( $>1.5 \mathrm{mg} / \mathrm{dl})$, functional status and type of surgery) are recommended for cardiac perioperative risk stratification in non-cardiac surgery $[11,12]$. The NSQIP model is presented as an interactive risk calculator (http://www.surgicalriskcalculator.com/miorcardiacarrest) and the risk can be calculated in a simple and accurate way.

Table 1. Surgical risk procedures and incidence of perioperative cardiac complications. ${ }^{*}$

\begin{tabular}{lll} 
Low risk $<1 \%$ & Intermediate risk $\mathbf{1 - 5} \%$ & High risk $>5 \%$ \\
Superficial surgery & Abdominal & Aortic and major vascular surgery \\
Brest & Carotid symptomatic & Open lower limb amputation \\
Dental & Peripheral arterial angioplasty & Duodeno-pancreatic surgery \\
Endocrine & Endovascular aneurysm repair & Liver resection, bile duct surgery \\
Eye & Head and neck surgery & Oesofagectomy \\
Reconstructive & Pulmonary & Repair of perforated bowel \\
Minor gynecological & Renal transplant & Adrenal resection \\
Minor orthopedic & Minor intratoracic & Total cistecomy \\
Minor urologic & Major neurologic & Pneumonectomy \\
& Major orthopedic & Pulmonary transplant \\
& Major urologic & Liver trasplant \\
\hline
\end{tabular}

* 30-day cardiovascular death and nonfatal miocardial infarction, patient comorbidities not considered. 


\section{Evaluate biomarkers}

Based on present data in the literature, assessment of serum biomarkers (troponin, B-type natriuretic peptide, etc.) for patients undergoing non-cardiac surgery cannot be proposed for routine use, but may be considered in high-risk patients (METs $\leq 4$ or with a revised cardiac risk index value $>1$ for vascular surgery, and $>2$ for non-vascular surgery), in order to obtain independent prognostic information for perioperative and late cardiac events [6].

\section{Decide if further noninvasive/invasive evaluation is needed}

Patient who are at low cardiac risk based on clinical features, functional status, and proposed low-risk surgery (Table 1) do not generally require further cardiac evaluation, and can be operated on safely without further delay. Additional preoperative testing is indicated in those patients at intermediate risk where functional status is poor or unclear. Patients considered at high-risk based on clinical features, poor functional status, undergoing high-risk surgery (Table 1) may benefit from further evaluation, and testing should be performed, mostly when it can change perioperative management.

If active cardiac disease is suspected, the patient should be referred to a cardiologist for assessment and possible treatment.
Active cardiac conditions that necessitate further evaluation and treatment are:

1) unstable coronary sindromes (unstable or severe angina; recent ( $<30$ days) myocardial infarction);

2) decompensated heart failure;

3) significant arrhytmias (high grade atrioventricular block; symptomatic ventricular arrhytmias; supraventricular arrhytmias with uncontrolled ventricular rate (>100 bpm); symptomatic bradycardia; newly recognized ventricular tachycardia);

4) severe valvular disease (aortic stenosis: mean pressure gradient $>40 \mathrm{mmHg}$, area $<1 \mathrm{~cm}^{2}$ or symptomatic; symptomatic mitral stenosis).

In seniors undergoing non-cardiac surgery, invasive cardiac evaluations (such as preoperative coronary angiography) can be indicated in patients considered at high risk based on noninvasive testing, angina unresponsive to adequate medical therapy, unstable angina, and proposed intermediate or high-risk surgery after equivocal noninvasive test results.

Individuals with more than 3 clinical risk factors, and extensive myocardial ischemia on preoperative stress imaging testing appear to have a high complication rate, even with effective medical therapy, and should be considered for invasive evaluation and coronary revascularization ("individualized management" on the algorithm).

When senior patients need to undergo a surgical non-cardiac procedure, the anesthesiologist should coordinate a multidisciplinary team (cardiologist, geriatrician, surgeon), for determining which patients benefit from cardiac testing, coronary artery revascularization, and cardiovascular therapy before surgery, according to the stepwise approach (algorithm) illustrated in Figure 1.

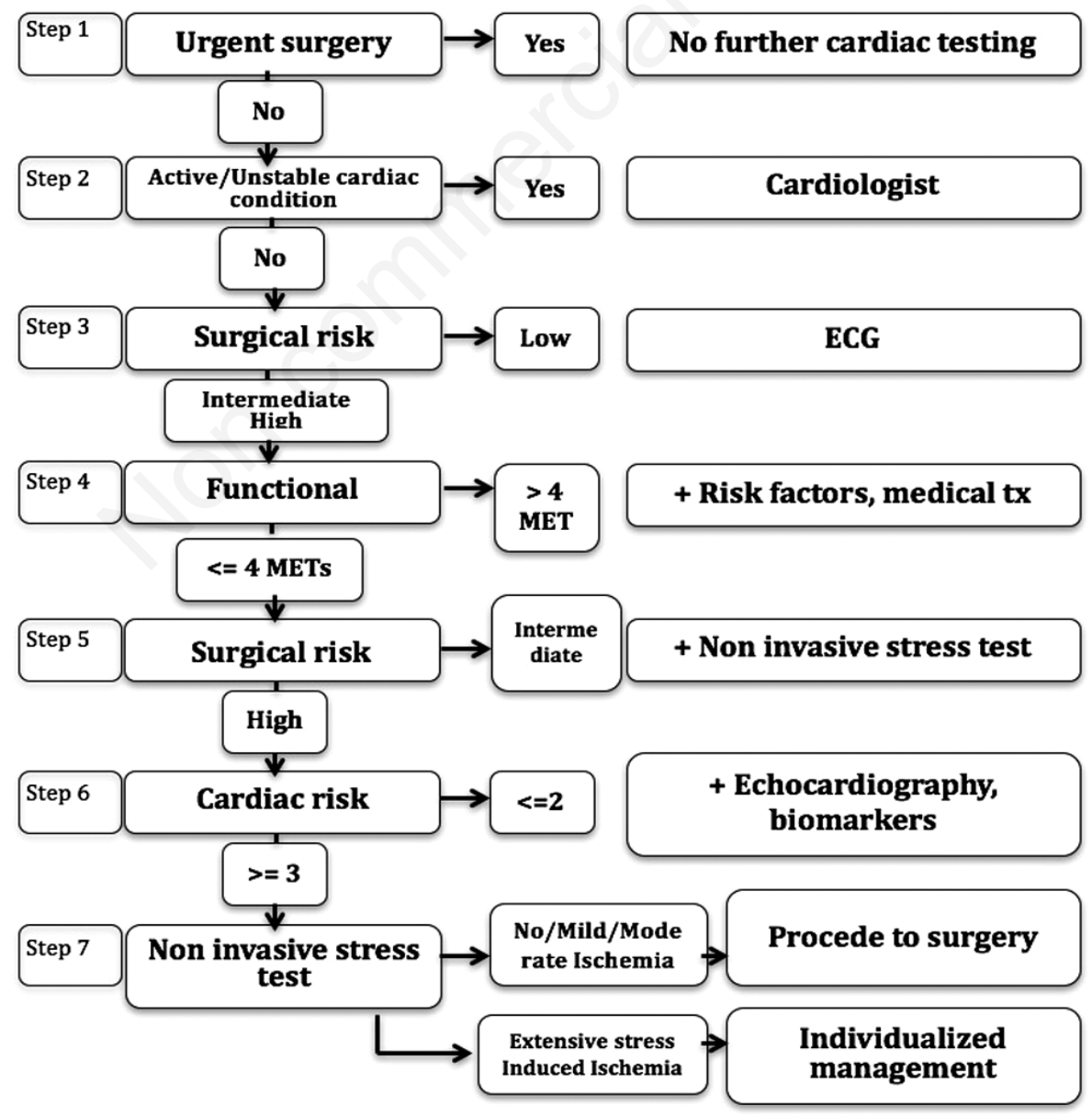

Figure 1. Algorithm for patients undergoing non-cardiac surgical procedure. 


\section{Perform postoperative surveillance}

Cardiac ischemia or miocardial infarction can be estimated on the basis of biomarkers elevation, new ECG abnormalities, hemodynamic instability, and quality and intensity of chest pain or other cardiac symptoms.

Patients who develop ST-elevation MI (STEMI) should be considered for urgent angiography and coronary reperfusion, whereas patients with non-STelevation MI (non STEMI) should undergo risk stratification after initial stabilization with intensive medical therapy. Individuals who develop heart failure after surgery should be evaluated and treated on the basis of the precipitating factors and/or underlying causes.

\section{Conclusions}

Comprehensive appropriate evaluation by a dedicated team, possibly including anesthesiologist, cardiologist, geriatrician and surgeon, may significantly improve periprocedural and long-term outcomes in senior patients undergoing non-cardiac surgery.

Currently, the prevention of cardiac problems relies on identification of the patients at risk, optimisation of the preoperative condition by modification when possible of underlying risk factors, optimisation of the perioperative medication, and adequate perioperative monitoring and measures to prevent myocardial ischaemia. These include adequate sedation and analgesia, adequate oxygenation, oxygen transport and ventilation, and if necessary additional cardiac medication [13].

\section{References}

1. Carroll K, Majeed A, Firth C, Gray J. Prevalence and management of coronary heart disease in primary care: population-based crosssectional study using a disease register. J Public Health Med 2003; 25:29-35

2. Rich MW, Chyun DA, Skolnick AH: Knowledge Gaps in Cardiovascular Care of the Older Adult Population: A Scientific Statement From the American Heart Association, American College of Cardiology, and American Geriatrics Society. Circulation. 2016; 133: 2103-22.
3. Biagioli B, Catena G, Clementi G, et al: Recommendations for the perioperative management of heart disease patients in non-cardiac surgery. Società Italiana di Anestesia, Analgesia, Rianimazione e Terapia Intensiva. Minerva Anestesiol. 2000; 66:85-104.

4. Poldermans D, Bax JJ, Boersma E, et al: Guidelines for pre-operative cardiac risk assessment and perioperative cardiac management in non-cardiac surgery: the Task Force for Preoperative Cardiac Risk Assessment and Perioperative Cardiac Management in Non-cardiac Surgery of the European Society of Cardiology (ESC) and endorsed by the European Society of Anaesthesiology (ESA). Eur J Anaesthesiol. 2010; 27:92-137.

5. De Hert S, Imberger G, Carlisle J, et al: Preoperative evaluation of the adult patient undergoing non-cardiac surgery: guidelines from the European Society of Anaesthesiology. Eur J Anaesthesiol. 2011; 28:684-722.

6. Kristensen SD, Knuuti J, Saraste A, et al. 2014 ESC/ESA Guidelines on non-cardiac surgery: cardiovascular assessment and management: The Joint Task Force on non-cardiac surgery: cardiovascular assessment and management of the European Society of Cardiology (ESC) and the European Society of Anaesthesiology (ESA). Eur J Anaesthesiol 2014;31:517-73.

7. Bettelli G. Preoperative evaluation in geriatric surgery: comorbidity, functional status and pharmacological history. Minerva Anestesiol 2011;77:637-46.

8. Nakhaie M, Tsai A. Preoperative assessment of geriatric patients. Anesthesiol Clin 2015;33:471-80.

9. Glance LG, Lustik SJ, Hannan EL, et al. The surgical mortality probability model: derivation and validation of a simple risk prediction rule for noncardiac surgery. Ann Surg 2012;255:696-702.

10. Tsiouris A, Horst HM, Paone G, et al. Preoperative risk stratification for thoracic surgery using the American College of Surgeons National Surgical Quality Improvement Program data set: functional status predicts morbidity and mortality. J Surg Res 2012;177:1-6.

11. Lee TH, Marcantonio ER, Mangione CM, et al. Derivation and prospective validation of a simple index for prediction of cardiac risk of major noncardiac surgery. Circulation 1999;100:1043-9.

12. Gupta PK, Gupta H, Sundaram A, et al. Development and validation of a risk calculator for prediction of cardiac risk after surgery. Circulation 2011;124:381-7.

13. Damen J, Hagemeijer JW, van den Broek L, et al. Prevention of perioperative cardiac complications in non-cardiac surgery: an evidence-based guideline. Ned Tijdschr Geneeskd 2008;52:2612-6. 